\title{
Percepção de familiares e profissionais de saúde sobre os cuidados no final da vida no âmbito da atenção primária à saúde
}

\author{
Perception of family members and health professionals \\ on end of life care within the scope of primary health care
}

Ana Helena Araújo Bomfim Queiroz ${ }^{1}$

Ricardo José Soares Pontes ${ }^{2}$

Ângela Maria Alves e Souza ${ }^{3}$

Thamy Braga Rodrigues ${ }^{3}$

\footnotetext{
${ }^{1}$ Faculdade Luciano Feijão. Av. Dom José 225, Centro. 62.010-290 Sobral CE. anahelenabqueiroz@ gmail.com

${ }^{2}$ Departamento de Saúde Comunitária, Faculdade de Medicina, Universidade Federal do Ceará.

${ }^{3}$ Departamento de Enfermagem-DENF, Universidade Federal do Ceará.
}

\begin{abstract}
The objective of this study is to reflect on care for people with terminal illnesses in primary health care. This study is relevant in the current context of demographic and epidemiological transition, in which there is an increase in the aging population and a rise in the prevalence of chronic degenerative diseases. By means of qualitative research with a critical hermeneutic focus, individual interviews were conducted with seven family members, three professionals from the Family Health Strategy and two professionals from the Home Care Program. The analysis established two empirical categories: end of life care from the perception of family members and that of health professionals. The results showed that, despite the humanization discourse, reflected in the prerogative of living the final moments of life with the family, a serious problem in the discontinuity of care is concealed. It was concluded that, despite the efforts, little is achieved in improving the quality of life for those who die from terminal diseases at home in the Unified Health System in municipalities distant from large centers.
\end{abstract}

Key words Palliative care, Primary Health Care, Home care
Resumo O objetivo deste estudo é refletir sobre os cuidados às pessoas com doenças em fase terminal na atenção primária à saúde (APS). Esta pesquisa torna-se relevante no contexto atual de transição demográfica e epidemiológica, na qual há um crescente envelhecimento populacional e um aumento da prevalência de doenças crônico-degenerativas. Por meio de pesquisa qualitativa sob o enfoque hermenêutico-crítico, foram realizadas entrevistas individuais com sete familiares, três profissionais da Estratégia Saúde da Família (ESF) e dois profissionais do Programa de Assistência Domiciliar. A análise ressaltou duas categorias empíricas: cuidados no final da vida na percepção dos familiares; e na dos profissionais de saúde. Os resultados evidenciaram que, apesar do discurso de humanização, refletida na prerrogativa de viver os últimos momentos de vida com a família, esconde-se um grave problema de descontinuidade dos cuidados. Concluiu-se que, apesar dos esforços, pouco se logra de melhoria na qualidade de vida de quem morre por uma doença em fase terminal no domicílio, sob os cuidados do Sistema Único de Saúde (SUS) em municípios distantes dos grandes centros.

Palavras-chave Cuidados paliativos, Atenção Primária à Saúde, Assistência domiciliar 


\section{Introdução}

O cenário sociossanitário do processo de transição demográfica e epidemiológica, caracterizado pelo crescente envelhecimento populacional e aumento da prevalência de doenças crônico-degenerativas (enfermidades cardiovasculares e neoplasias), colocou em evidência os cuidados no final da vida, demandando a reorganização dos serviços de saúde ${ }^{1}$.

Simultaneamente, a "medicalização" social, decorrente do intenso avanço tecnológico da assistência à saúde e da crescente interferência da Medicina no cotidiano individual, transformou a morte, de um fenômeno humano antes compartilhado pela família e pela comunidade, em um acontecimento técnico próprio dos hospitais. Neste contexto, o movimento Hospice, assim como outros de reivindicação dos direitos dos pacientes, originários nos EUA, puseram em pauta os cuidados aos pacientes com doenças terminais e o conceito de "boa morte", como fundamental para a assistência no final da vida ${ }^{2}$.

A expressão "paciente terminal" é utilizada para designar pessoas com patologias de prognóstico reservado, mesmo em fase de diagnóstico e tratamento ${ }^{3}$. A grande questão no seu uso é a estigmatização da pessoa com o rótulo de "terminal”, muitas vezes responsável por um desinvestimento médico e desistência de cuidados por parte de profissionais e familiares. Ao contrário, os momentos finais da vida exigem um cuidado humanizado e tecnicamente adequado, priorizando o manejo dos sintomas e a qualidade de vida. Isso exige uma nova postura assistencial e investimentos na formação dos profissionais de saúde ${ }^{4}$.

Apesar da ampliação dos cuidados paliativos como modalidade de atenção em todo o mundo, estima-se que cerca de 20 milhões de pessoas ainda morrem sem acesso à atenção que poderia minimizar seu sofrimento e os sintomas de suas doenças ${ }^{5}$. No Brasil, há carência de uma política nacional que articule ações para o final da vida, dentro do modelo de cuidado paliativo, que significa valorizar o controle dos sintomas, a abordagem integral e multidisciplinar, envolvendo o doente e seu grupo familiar, desde o diagnóstico até o luto ${ }^{6}$. Nesse sentido, alguns desafios a serem enfrentados são a dificuldade no diagnóstico da "terminalidade", o uso de intervenções prolongadas, em detrimento de condutas de alívio do sofrimento, além da descontinuidade do tratamento na transição dos cuidados curativos para os paliativos, acarretando em abandono e isolamento dos doentes e seus familiares ${ }^{6}$.
Os cuidados paliativos podem ser desenvolvidos no contexto ambulatorial, de internação hospitalar e no domicílio. Como os estudos em geral enfocam o cuidado no hospital ou Hospice, compreender como esse tipo de atenção poderia ser desenvolvido no domicílio e por profissionais da atenção primária certamente ampliaria as perspectivas dos pesquisadores e contribuiria para suscitar mais ideias no que concerne ao tema.

A atenção aos doentes terminais e o controle da dor crônica no contexto da atenção básica, formalmente, incorporam-se ao conjunto de responsabilidades das equipes da atenção primária/Estratégia Saúde da Família (ESF) ${ }^{7,8}$. Conforme diretrizes recentes ${ }^{9}$, a atenção básica é responsável por ordenar o cuidado e a ação territorial, em articulação com as equipes multiprofissional de atenção domiciliar (EMAD).

A assistência domiciliar tem um papel preponderante nesse âmbito, sendo justificada pelo elevado grau de humanização que pode propiciar, pois envolve a família tanto nos cuidados como no amparo afetivo ao paciente, reduz complicações decorrentes de longas internações hospitalares e diminui os custos das tecnologias dos doentes hospitalizados. A incorporação de mais saberes e práticas de cuidado ao processo de trabalho das equipes de Saúde da Família/Atenção Básica, bem como a articulação dessa tarefa aos demais serviços, contribuem de modo significativo para ampliar a integralidade da atenção.

Objetiva-se, enfim, identificar e compreender as percepções de cuidado e as principais dificuldades enfrentadas pelos profissionais de saúde e familiares no acompanhamento de pessoas com doença em quadro terminal no domicílio, visando à reflexão sobre o potencial da atenção primária à saúde na garantia de uma morte digna.

\section{Percurso metodológico}

Realizou-se um estudo de natureza qualitativa, na vertente hermenêutica crítica ${ }^{10}$, onde a interpretação constitui-se um movimento dialético, comprometido com o resgate crítico do contexto do fenômeno estudado, no caso, a morte em domicílio. Destaca-se, ainda, a relevância das pesquisas qualitativas na formulação e avaliação de políticas públicas em saúde no Brasil ${ }^{11}$.

O estudo foi realizado em município de médio porte do Nordeste brasileiro (Sobral, região norte do estado do Ceará), referência econômica e de serviços para os demais municípios da região, com estimativa de 193.134 habitantes $^{12}$. É 
sede de macrorregião de saúde, que engloba 55 outros municípios, abrangendo uma população de aproximadamente 1.600 .000 habitantes.

Tomando como referencia a proposta operativa de articulação da hermenêutica e da dialética como práxis interpretativa ${ }^{13}$, considerou-se como primeiro momento interpretativo a fase exploratória da pesquisa, que proporcionou maior inserção na realidade local e compreensão do funcionamento do sistema de saúde e das condições de vida da população.

$\mathrm{Na}$ fase exploratória (2009), analisou-se a mortalidade e suas causas a partir do Sistema de Informação de Mortalidade (SIM) do Município, observando-se elevadas taxas de óbitos classificados como decorrentes de "causas mal definidas", indicador indireto da magnitude de desfechos que, em parte, ocorrem nos domicílios ${ }^{14}$. Avaliou-se, a seguir, a incidência de óbitos tendo por causas as doenças do aparelho circulatório e as neoplasias, ocorridas no ambiente domiciliar, observando-se a inexistência de registros sobre tais eventos - ou as respectivas condutas e cuidados ofertados - nas unidades de saúde da família. Essa avaliação permitiu concluir que seria relevante, para o sistema de saúde do Município, compreender a maneira como os doentes com diagnóstico firmado, e que faleceram em casa, foram acompanhados até o momento de sua morte.

Investigaram-se, então, as declarações de óbito (DO) do período de janeiro a julho de 2010, de pessoas cujo desenlace havia ocorrido no domicílio e que apresentavam causa básica de óbito definida. Nesse intervalo de tempo, houve 160 óbitos em domicílio, dentre os quais se identificaram 44 com causas definidas registradas na DO, constituindo-se a fonte inicial de sujeitos elegíveis para o estudo. Estes casos foram organizados por nome, idade, sexo, causa básica, bairro, ocupação, médico responsável e mês do óbito. Demarcou-se o espaço territorial de pesquisa, englobando-se os territórios de abrangência da ESF na sede (11) e nos distritos (4) relacionados aos 44 óbitos domiciliares. Os gerentes das unidades de saúde de onde provieram os óbitos foram contatados para apoiar na identificação dos familiares e na articulação com os profissionais, visando à realização das entrevistas individuais. Após as primeiras interlocuções, as equipes informaram sobre outras famílias que estavam vivenciando, naquele momento, a situação de terminalidade de um familiar, tornando-as também elegíveis como sujeitos a serem incluídos na amostragem qualitativa.

Relevante critério de seleção em amostras qualitativas por casos múltiplos é a diversificação; ou seja, a busca de variabilidade nas circunstâncias de observação e nos informantes, considerando os objetivos da pesquisa ${ }^{15}$. Assim, utilizou-se como critério de seleção dos participantes a variabilidade quanto ao território (Sede e Distrito), as categorias profissionais (enfermeiro e médico, fisioterapeuta, terapeuta ocupacional) e a ocorrência do óbito da pessoa com doença em estágio terminal (ter ocorrido ou não). Considerou-se também o critério de saturação para a quantidade ideal de participantes. A saturação empírica ocorre quando o pesquisador julga que os últimos documentos, entrevistas ou observações não trazem informações suficientemente novas para justificar a ampliação da coleta dos dados ${ }^{15}$.

Foram selecionados como participantes da pesquisa quatro familiares de pessoas que morreram no domicilio (janeiro a julho de 2010), três familiares de pessoas com doença em decurso terminal acompanhadas pela ESF, três profissionais de saúde da ESF e dois do Programa de Assistência Domiciliar do Município, totalizando 12 "informantes-chave". Considerou-se como critério de inclusão para familiar, o cuidador com grau de parentesco e que acompanhou mais diretamente a pessoa com a doença em período terminal, em suas necessidades diárias. Realizou-se entrevista individual com os familiares no domicílio, em espaço reservado. A questão norteadora para os familiares foi "como é cuidar de um parente com uma doença em estágio terminal no domicílio?” Os profissionais de saúde também foram entrevistados em salas reservadas. A pergunta-guia aos profissionais de saúde foi: "como é cuidar de uma pessoa com uma doença em estágio terminal no domicílio?" As entrevistas foram conduzidas, gravadas e transcritas pelo pesquisador principal.

Dos sete familiares, todas as entrevistadas eram mulheres, com idade média de 39,2 anos, variando de 29 a 60 anos; três eram filhas, duas irmãs, uma neta e uma cunhada dos sujeitos de referência. Não se encontrou nenhum do sexo masculino, o que reafirma o papel cultural da mulher como principal cuidadora da família ${ }^{16}$. Dentre os cinco profissionais de saúde entrevistados, três compunham a equipe de Saúde da Família (dois enfermeiros e um médico) e dois faziam parte da equipe do Programa de Assistência Domiciliar (um terapeuta ocupacional e um fisioterapeuta). Os profissionais da ESF tinham um tempo de atuação na função de seis a oito anos, portanto com larga experiência nas ações da atenção primária.

$\mathrm{O}$ segundo momento interpretativo corresponde o encontro com os fatos empíricos ${ }^{13}$. Após 
leitura transversal do conteúdo obtido nas entrevistas, foram identificadas categorias representativas da narrativa dos informantes. Analisar e interpretar material qualitativo sob a vertente hermenêutico-dialética deve explorar o contexto dos entrevistados, compreender seus relatos "neles mesmos" e buscar as diferenças e semelhanças entre seu contexto e aquele dos entrevistados, levando em consideração que as relações sociais são dinâmicas e contraditórias entre classes, grupos e culturas ${ }^{17}$.

Este estudo foi aprovado pelo Comité de Ética em Pesquisa da Universidade Estadual Vale do Acaraú (UVA). Após esclarecimento, os informantes que aceitaram participar da pesquisa assinaram o Termo de Consentimento Livre Esclarecido (TCLE).

\section{Resultados e discussão}

\section{Cuidados no final da vida: percepções dos familiares}

Considera-se que os doentes elegíveis para os cuidados paliativos são pessoas com enfermidades crônicas, evolutivas e progressivas, com prognóstico de vida supostamente encurtado a meses ou ano. Em situações de doenças degenerativas, como as demências, o que é levado em consideração é o momento de constatação da alta dependência para as atividades diárias, o que pode se prolongar por mais de um ano ${ }^{18}$.

O significado atribuído ao cuidado no final da vida no domicílio compreende realizar ações práticas de suporte cotidiano como higiene, alimentação, medicamentos, verificar a pressão, a glicemia; mas também, em outro sentido de cuidar, garantir o conforto e oferecer amor, na percepção de familiares cuidadores. Cuidar significa, então, auxiliar no que o outro não consegue fazer por si mesmo, oferecer resposta emocional de apoio e estar junto. Tal atitude muitas vezes é difícil, sofrida, exige paciência e renúncia. Constitui-se uma função de muita responsabilidade, no entanto contraditória afetivamente, sendo necessário "ter pulso e ser mansa", mas, principalmente, respeitar o papel e o lugar do doente na dinâmica familiar:

[...] porque é difícil. Porque eu tenho que respeitar ela como minha mãe primeiro. [...] Tem que ter pulso. Tem que ser mansa, mas tem que ter pulso. (Entrevistado 02- familiar).

Esse cuidar também tem o sentido de dever e gratidão na esfera das relações intrafamiliares, por ter sido o cuidador, ele mesmo, cuidado em algum momento da vida, significando a possibilidade de oferecer retribuição afetiva enquanto o familiar estiver vivo:

Cuido porque é minha mãe e eu devo a ela minha vida. Teve o tempo que ela gastou comigo, os nove meses, as doenças. Tem a hora de a gente retribuir. (Entrevistado 02- familiar).

As relações de cuidado domiciliar são permeadas por sentimentos de gratidão, afeto, amor, retribuição, medos, culpas, conflitos e adoecimento físico ou emocional do cuidador. No entanto, quando a escolha do familiar para cuidar de alguém no final da vida está baseada no sentimento de obrigação moral, torna-se necessário um esforço maior para ressignificar a relação e desenvolver estratégias para lidar com a finitude ${ }^{19}$.

O cuidar no domicílio exige uma nova dinâmica de trabalho e de organização da família. Essas são dificuldades recorrentes nas narrativas dos cuidadores, uma vez que nem sempre os demais membros da família estão disponíveis para compartilhar as tarefas. Na maioria dos casos, o cuidador principal fica sobrecarregado. O próprio nunca se considera suficientemente bom e dedicado ${ }^{20}$. Quando possível, o rodízio entre os cuidadores é importante estratégia para reduzir o desgaste físico e emocional de quem fica mais próximo e se dedica mais. Como o doente se torna o foco da atenção, o cuidador se torna mais exposto ao risco de cansaço e fadiga, sobretudo quando a morte do familiar se torna iminente:

Eu estava me acabando, eu também já tenho problema de pressão. Já estou com começo de diabetes também. A diabetes chegou de uns tempos para cá porque eu não tinha isso. (Entrevistado 03 - Familiar).

O suporte nas necessidades domiciliares cotidianas relativas ao manejo adequado do familiar demanda apoio mais especializado, quase nunca suprido pelos serviços e profissionais, quais sejam, na percepção dos cuidadores: falta de assistência médica e de apoio de profissionais de saúde no domicílio para prescrever medicamentos, atender as urgências e as necessidades de fisioterapia; falta de recursos e suporte como alimentação, medicamentos de alto custo e de transporte quando precisam levar seu ente querido aos serviços de saúde, necessitando para isso da ajuda de pessoas da família e de vizinhos; além da ausência de suporte psicológico em relação ao estigma do câncer, quando o familiar sofre essa enfermidade. Esse estigma se reforça, seguido pelo abandono institucional, sobretudo se o diagnóstico é tardio, dificultando o tratamento e reduzindo as chances de cura. 
O que eu mais gostaria é que tivesse pelo menos um médico. Porque no posto só tem as enfermeiras e não tem médico. Um médico mesmo para dizer, por exemplo, ela tem uma ferida aqui, nos quartos, que já está muito assim, grande. A gente não pode dar medicamento nenhum para ela tomar porque tem que ser passado, no caso dela, com receita médica. E não tem quem dê uma receita. A gente tem como comprar o remédio, mas não tem um médico para passar (Entrevistado 06 - Familiar).

Conviver com o familiar doente em casa, além do conforto psicológico para o próprio doente terminal, permite introduzir outra lógica no âmbito da cultura familiar, facilitando a experiência existencial de lidar melhor com o luto que, assim, ocorrerá de modo processual, proporcionando mais conforto espiritual e melhor qualidade de vida aos sobreviventes. Considere-se, entretanto, que nem sempre é possível cuidar no domicílio, sendo necessário levar em conta o estado do doente, questões ambientais e de suporte familiar. A decisão sobre esse tipo de cuidado é complexa, porquanto pode exigir transformações de ordem prática, como adaptação da casa, estabelecimento de rotinas e definição dos cuidadores. A manutenção da rotina cotidiana acarreta custos elevados, principalmente, quando ela se estende por tempo prolongado:

No hospital, vocêtem que arrumar uma pessoa de qualquer maneira. Eu aqui tinha menino para mandar para o colégio, tinha o que fazer, tinha as coisas dela para cuidar. (Entrevistado 08 - Familiar).

É importante que o profissional da ESF tenha compreensão da dinâmica da família do enfermo e do papel que o doente aí desempenha. Frequentemente, a casa onde um enfermo ficará é palco de diferentes arranjos e relações intrafamiliares que as transformações do casamento intensificaram e diversificaram em anos recentes. Há lares onde vivem crianças e jovens de vários casamentos, netos e avós. A família, se bem abordada e trabalhada, pode ser considerada um agente terapêutico eficaz, uma vez que se configura como elo entre o paciente e a equipe de saúde; em caso contrário, pode influenciar negativamente na evolução da pessoa doente ${ }^{21}$. Assim, representa uma importante tarefa auxiliar a família na tomada de decisão de quem será o cuidador e como se reorganizará a casa, considerando-se as várias circunstâncias.

Uma vez decidido que o doente ficará em seu domicílio, é preciso fornecer a assistência requerida, acompanhar a organização do cuidado e observar as dificuldades dos familiares, visando dar suporte ao doente e ao cuidador. O acompanhamento longitudinal no território, característica da ESF, favorece esse tipo de abordagem que, todavia, não ocorre de forma automática: precisa ser pensada e se tornar uma ação de apoio da equipe.

\section{Cuidados no final da vida: percepções dos profissionais de saúde}

O conceito de qualidade de vida, motivador do movimento Hospice, por ser multidimensional, engloba os aspectos físicos, sociais, psicológicos e espirituais. A atuação com vistas à qualidade de vida exige o controle dos sintomas, a minoração do sofrimento, a busca do conforto espiritual e o fortalecimento dos vínculos famili$\operatorname{ares}^{22}$. Por conter, porém, um elevado grau de subjetividade, o conceito de qualidade de vida é percebido de forma diferente pelo doente, pelos familiares e pelos profissionais de saúde, o que torna mais difícil a sua operacionalização no cotidiano $^{6}$. O compromisso com a qualidade de vida dos doentes e cuidadores familiares supõe uma formação específica por parte dos profissionais e a organização de serviços adequados para atuar nessas situações. Cuidar no domicílio exige articulação de conhecimentos técnicos e uma atitude de compaixão-empatia para com o outro. A percepção do profissional considera que sua atuação deve estar centrada na orientação e no apoio ao doente e à família, contribuindo para uma melhor qualidade da vida em termo:

A gente trabalha em cima da qualidade de vida. Para que esses últimos dias, esses últimos momentos sejam o mais agradável possível para ele. Para que ele não fique pensando e com medo. (Entrevistado 11 - Profissional de saúde).

Várias dimensões de formação, que extrapolam o campo da técnica, constituem os aspectos contextuais de maior dificuldade no ato de cuidado qualificado aos doentes terminais no domicílio. Destacam-se, na percepção dos profissionais, a própria limitação emocional para lidar com a morte ou com a impossibilidade de cura, além da inadequada formação para comunicar o diagnóstico e relacionar-se com a família do paciente. Os profissionais de saúde reconhecem que cuidar do doente em fase terminal no domicílio exige preparação para lidar com os próprios sentimentos. No cotidiano de trabalho surgem situações que provocam conflitos, por ser de difícil resolução, produzindo sentimentos de impotência, frustração e revolta. O fato de não conseguir evitar a morte leva a pensar na 
própria finitude, experiência esta, na certa, bastante dolorosa. As situações de muito sofrimento do doente, de pacientes jovens ou de forte vínculo da equipe com a pessoa doente, também podem marcar os profissionais ${ }^{23}$ :

A gente no início, quando a gente começa a atender um paciente terminal, a gente se sente sensibilizado. Até porque a gente sabe que aquele paciente não vai durar muito, a gente está ali, mas não vai ter um objetivo maior. Às vezes, no início eu me sentia mal porque eu estava ali, mas eu não ia dar uma qualidade... eu ia dar uma qualidade, mas no fundo no fundo, eu não poderia ajudar ele como eu queria. (Entrevistado 12 - profissional de saúde).

Como a formação técnica está baseada no paradigma da cura $^{24}$, o profissional de saúde, mesmo que considere atuar na perspectiva da qualidade de vida, ainda se sente vulnerável perante a ocorrência da morte. Por consequência, tornase importante favorecer a coesão grupal e estimular o diálogo entre os profissionais, proporcionando apoio, em grupo ou individualmente, e estimulando o autoconhecimento. Em geral, o tema da morte não é discutido na graduação e, no trabalho prático, quase sempre é desqualificado ${ }^{25}$.

Os profissionais consideram que cuidar no domicílio exige formação técnica específica, de que não são possuidores. Ressentem-se da falta de conhecimentos e habilidades para realizar tal ação fora dos espaços institucionais onde normalmente atuam. Consideram ser importante reconhecer aquilo que é possível realizar no domicílio e o que deve ser encaminhado a algum centro de atenção de maior complexidade. Assim, apresentam dificuldades em realizar procedimentos técnicos no espaço do domicílio, no contexto da ausência de retaguarda dos serviços especializados, considerando a precária organização desse tipo de cuidado domiciliar frente a tantas outras demandas a que estão submetidos:

Porque você sabe que como tem a morte, também tem a mortalidade, também tem as outras coisas, tem as visitas, tem as gestantes, tem noutras coisas. Mas acredito que a gente como PSF pode fazer essa visita, dar esse apoio... (Entrevistado 01 - Profissional de saúde).

Do ponto de vista técnico e organizacional, os serviços de atenção primária, para desempenhar o papel de coordenação do cuidado, necessitariam de um quadro técnico-profissional com maior competência para realizar alguns procedimentos importantes no domicílio, além de dispor de recursos materiais básicos, tais como medicamentos e outros insumos necessários à melhoraria do conforto do doente. A inexistência dessas condições gera problemas de organização do processo de trabalho, servindo de discurso justificador para a exclusão da atenção continuada ao paciente em fase terminal no contexto de tantas atividades:

Existem vários pacientes em estado terminal, só que a gente não tem aquele acompanhamento tão de perto. A gente vai por alguma intercorrência que acontece, então a gente vai e faz a visita domiciliar e pronto. Mas aquele acompanhamento mais de perto, geralmente não é feito [...] (Entrevistado 07 - Profissional de saúde 07).

Um dos principais entraves à otimização dos cuidados paliativos é a falta de uma cultura de cuidado compartilhado e de corresponsabilização entre os serviços de saúde dos diferentes pontos da rede, gerando informações contraditórias fornecidas por profissionais de saúde e outros funcionários, não familiarizados com as questões relacionadas à morte ${ }^{26}$.

A organização do processo de trabalho e da articulação da rede assistencial nas políticas públicas de assistência domiciliar, mesmo entre ações especificamente voltadas para esse fim (como o Programa de Assistência Domiciliar e a ESF), encontra dificuldades de operacionalização tanto na identificação e no encaminhamento dos doentes com perfil para esse tipo de atendimento, como na ausência de compartilhamento recíproco de atividades. A existência de uma grande e variada demanda espontânea na atenção primária, muitas vezes, impede o fornecimento de uma assistência de qualidade aos enfermos em situação de terminalidade e aos cuidadores. $\mathrm{Na}$ relação da ESF com o hospital - aspecto comum a familiares e profissionais - prevalece a percepção de que, após o diagnóstico de uma doença terminal, o hospital encaminha os doentes para a casa porque não há mais o que ser feito, sem comunicação prévia aos profissionais responsáveis pelo acompanhamento domiciliar:

A gente levava ele para internar, passava 1 ou 2 dias e ele voltava para casa porque não tinha jeito. [...] Ninguém pedia para voltar. Eles que mandavam. (Entrevistado 03 - Familiar).

Grande parte dos hospitais brasileiros não possui diretrizes de como cuidar de pessoas com doenças em fase irreversível e nem informações sistematizadas de como os pacientes, a família e os profissionais lidam com os últimos momentos.

Os cuidados paliativos a um doente envolvem diferentes partes do sistema de saúde e, frequentemente, se faz necessário um tipo de colaboração ou de assistência compartilhada. Os 
cuidados no final da vida significam um campo de conhecimentos e práticas que exige atuação multiprofissional. Torna-se, pois, fundamental a organização da assistência com equipe mínima e em articulação com os demais profissionais atuantes no domicílio, como o Núcleo de Apoio à Saúde da família (NASF) e o serviço de atenção domiciliar:

O hospital responsabiliza o PSF, o PSF responsabiliza o hospital. Então fica esse jogo de empurra e a família fica no meio sem saber o que fazer. Geralmente, a família vai culpar o PSF porque está mais próximo. Você sempre vai culpar quem está mais próximo de você. E porque eles ouvem mais o pessoal do hospital. Se o pessoal do hospital disse que é o PSF que tem que fazer, isso vale muito mais para eles do que a gente do PSF dizer que é o hospital que tem que fazer. (Entrevistado 07 - Profissional de saúde).

O papel da ESF nos cuidados ao final da vida atualmente é muito restrito, estando limitado a procedimentos de retaguarda, como articular, junto à rede de serviços, as internações e outros procedimentos necessários ao bem-estar do doente; em providenciar transporte para o doente permitindo o acesso a serviços de maior complexidade; em identificar as necessidades, dispensar medicamentos e outros recursos, como sonda e colchão d'água; em orientar e confortar a família; e, quando do falecimento, fornecer o atestado de óbito ${ }^{27}$ :

Acho que ele pode tratar da assistência a família, dar esse feedback do que está sentindo, do que está precisando. Está precisando de uma internação, faz a transferência dignamente, se comunicar com uma pessoa do hospital. Se for um curativo, pode dar orientações. Acho que isso o PSF pode fazer. A questão do cuidado, da escara, da mudança de decúbito, agendar... (Entrevistado 01 - Profissional de saúde).

A atenção primária, considerados seus atri$\operatorname{butos}^{28}$, representaria a possibilidade de um cuidado mais próximo da pessoa e de sua família, com grande potencial de estabelecimento de vínculo de confiança e de acompanhamento humanizado no fenômeno de morrer. Com a implantação do NASF, outros profissionais passaram a compor a equipe da ESF, como assistentes sociais, psicólogos, fisioterapeutas, terapeutas ocupacionais, favorecendo a atenção integral e humanizada, inclusive a terminal, no próprio domicílio.

\section{Considerações finais}

A partir da década de 1960, no contexto internacional, passaram a receber maior atenção as discussões a respeito da morte e do processo de morrer humanizado, por meio de movimentos de ideias e práticas como o denominado Hospice.

No Brasil, sob essa inspiração, principalmente nos grandes centros urbanos, existem diversos programas de cuidados paliativos vinculados aos hospitais, tendo a assistência domiciliar como modalidade de intervenção, além do serviço ambulatorial e de internação hospitalar tradicionalmente conhecidos.

Contrariamente, principalmente nas localidades distantes dos grandes centros populacionais urbanos, os pacientes em processos patológicos irreversíveis ou terminais, sofrem descontinuidade no processo de cuidado após a alta hospitalar. Esse problema enseja uma discussão importante sobre a contribuição da atenção primária e da ESF nos cuidados ao final da vida.

Dada a atribuição da ESF de acompanhar as pessoas e as famílias em suas necessidades de saúde no território, torna-se fundamental incrementar o potencial técnico e humanístico de sua equipe, direcionando-a também à prestação de cuidados no final da vida. O diferencial da ESF, frente a outros serviços, seria a possibilidade de estabelecimento de vínculo assistencial ao longo do tempo e, por consequência, do cuidado mais humanizado.

Existem, todavia, diversas dificuldades para a realização dessa ação. Isso ocorre tanto na organização como na operacionalização, na destinação de recursos e materiais, como na adequada assistência domiciliar. Igualmente, a formação voltada somente para a cura afasta os profissionais dos momentos finais, da morte e da vivência do luto.

O discurso da humanização dos cuidados, refletida no direito humanitário de viver os últimos momentos de vida com a família, pode esconder um grave problema de descontinuidade terapêutica e assistencial. Ao identificar a impossibilidade de cura, o doente é encaminhado para casa, sem a devida referência à ESF ou a outro serviço de assistência domiciliar. Mesmo com os esforços da família e dos profissionais do SUS, pouco se logrou melhorar na qualidade de vida de quem morre por uma doença em estágio terminal no domicílio, em realidades desprovidas de recursos humanos, gerenciais e materiais.

Os principais desafios da assistência domiciliar são estabelecer a parceria com a família, apren- 
der a trabalhar em conjunto com a comunidade, estabelecer a rede de apoio social e ter retaguarda efetiva dos demais serviços que cuidam dos pacientes nos diversos níveis de atenção à saúde. Transladar um paciente de um hospital para a casa, sobretudo, nos municípios afastados dos grandes centros, sem uma organização que dê apoio efetivo a ele e a sua família, pode significar privá-lo de uma atenção necessária a que tem direito.

Também é importante lembrar que os cuidados paliativos não devem ser considerados apenas no final da vida, uma vez que são plenamente compatíveis com cuidados tanto preventivos como curativos. Desse modo, é importante refletir sobre a necessidade da adoção de medidas preventivas de controle dos sintomas ao longo do curso das doenças crônicas, não esperando o doente ser considerado "terminal" pelos profissionais de saúde para adotá-las.

Finalmente, a expressão "paciente terminal", do ponto de vista psicossocial, pode favorecer a interpretação de que não há nada a ser feito, desobrigando os serviços de saúde de maior complexidade em suas responsabilidades assistenciais, levando o doente e sua família ao abandono e à desassistência, travestido do rótulo de cuidado domiciliar. Embora tal expressão produza confusões, ainda não há outra que a substitua adequadamente, uma vez que a denominação alternativa, paciente fora de possibilidades terapêuticas (FPT), também proporciona interpretações errôneas, vinculando necessariamente o vocábulo terapêutico ${ }^{23}$ ao termo cura.

\section{Colaboradores}

AHAB Queiroz trabalhou na concepção e redação final deste artigo; RJS Pontes orientou o trabalho original e participou da revisão do presente artigo; AMA Souza e TB Rodrigues contribuíram na revisão crítica. 


\section{Referências}

1. Floriani CA, Schramm FR. Desafios morais e operacionais da inclusão dos cuidados paliativos na rede de atenção básica. Cad Saude Publica 2007; 23(9):2072-2080.

2. Menezes RA. Tecnologia e "Morte Natural": o Morrer na Contemporaneidade. Physis 2003; 13(2): 129-147.

3. Kovács MJ. Educação para a Morte. Temas e Reflexões. São Paulo: Casa do Psicólogo; 2003.

4. Rego S, Palácios M. A finitude humana e a saúde pública. Cad Saude Publica 2006; 22(8):1755-1760.

5. Lima RAG. Cuidados Paliativos: desafios do sistema de saúde. Rev. Latino-Am. Enfermagem [periódico na internet]. 2011 Mar-Abr [acessado $2013 \mathrm{mar}$ 29]; 19(2): [2 telas]. Disponível em: http://www. scielo.br/pdf/rlae/v19n2/pt_01.pdf

6. Floriani CA. Moderno movimento hospice: fundamentos, crenças e contradições em busca da boa morte [dissertação]. Rio de Janeiro: Escola Nacional de Saúde Pública Sergio Arouca; 2009.

7. Brasil. Ministério da Saúde (MS). Agência Nacional de Vigilância Sanitária. Determina que a Notificação de Receita "A" não será exigida para dispensação de medicamentos à base das substâncias morfina, metadona e codeína, ou de seus sais, a pacientes em tratamento ambulatorial, cadastrados no Programa Nacional de Assistência à Dor e Cuidados Paliativos, do Sistema Único de Saúde e dá outras providências. Resolução RDC no 202, de 18 de julho de 2002. Brasília: Ministério da Saúde de 2002. Diário oficial da União 2002; 22 jul.

8. Brasil. Ministério da Saúde (MS). Portaria no 2.439/ GM de 8 de dezembro de 2005. Institui a Política Nacional de Atenção Oncológica: Promoção, Prevenção, Diagnóstico, Tratamento, Reabilitação e Cuidados Paliativos, a ser implantada em todas as unidades federadas, respeitadas as competências das três esferas de gestão. Brasília: Diário oficial da União 2005; 09 dez.

9. Brasil. Ministério da Saúde (MS). Portaria no 963/ GM de 27 de maio de 2013. Redefine a Atenção Domiciliar no âmbito do Sistema Único de Saúde (SUS). Diário oficial da União 2013; 07 jun.

10. Ayres JRCM. Hermenêutica e humanização das práticas de saúde. Cien Saude Colet 2005; 10(3):549560.

11. Bosi MLM. Pesquisa qualitativa em saúde coletiva: aportes aos sistemas de saúde. Cien Saude Colet 2012; 17(3):572-572.

12. Brasil. Ministério do Planejamento, Orçamento e Gestão. Instituto Brasileiro de Geografia e Estatística (IBGE). Contagem Populacional. [acessado 2013 jul 15]. Disponível em: http://www.ibge.gov.br/ home/estatistica/populacao/estimativa2012/ estimativa_dou.shtm

13. Minayo MCS. O Desafio do Conhecimento. 9a Edição. São Paulo, Rio de Janeiro: Hucitec, Abrasco; 2006.

14. Jorge MHM, Laurenti R, Gotlieb SLD. Análise da qualidade das estatísticas vitais brasileiras: a experiência de implantação do SIM e do SINASC. Cien Saude Colet 2007; 12(3):643-654.
15. Pires AP. Amostragem e Pesquisa Qualitativa: Ensaio Teórico e Metodológico. In: Poupart J, Deslauries J, Groulx L, Laperriere A, Meyer R, Pires AP, organizadores. A pesquisa qualitativa: enfoques epistemológicos e metodológicos. Petrópolis, RJ: Vozes; 2008. p. 154-211.

16. Bicalho CS, Lacerda MR, Catafesta F. Refletindo sobre quem é o cuidador familiar. Cogitare Enferm 2008; 13(1):118-123.

17. Minayo MCS. Hermenêutica-Dialética como Caminho do Pensamento Social. In: Minayo MCS, Deslandes SF, organizadores. Caminhos do pensamento: epistemologia e método. Rio de Janeiro: Fiocruz; 2002. p. 83-107.

18. Maciel MGS. Definições e Princípios. In: Oliveira RA, coordenador. Cuidado paliativo. São Paulo: Conselho Regional de Medicina do Estado de São Paulo; 2008. p. 16-32.

19. Fratezi FR, Gutierrez BAO. Cuidador familiar de idosos em cuidados paliativos: o processo de morrer no domicílio. Cien Saude Colet 2011; 16(7):3241-3248.

20. Ferreira NMLA, Souza CLB, Stuchi Z. Cuidados paliativos e família. Rev. Ciênc. Méd. 2008; 17(1):33-42.

21. Yoni TD. La familia como agente terapéutico en los cuidados paliativos. Medisan 2011; 15(2):197-203.

22. Melo AGC, Caponero R. Cuidados Paliativos: abordagem contínua e integral. In: Franklin SS, organizador. Cuidados Paliativos: discutindo a vida, a morte e o morrer. São Paulo: Atheneu; 2009. p. 257-267.

23. Kovács MJ. Desenvolvimento da Tanatologia: estudos sobre a morte e o morrer. Paidéia 2008; 18(41):457-468.

24. Pessini L. Bioética e cuidados paliativos: alguns desafios do cotidiano aos grandes dilemas. In: Pimenta CAM, Mota DDCF, Cruz DALM, organizadores. Dor e cuidados paliativos: Enfermagem, medicina e psicologia. Barueri: Manole; 2006. p. 45-66.

25. Schiliemann AL. Aprendendo a lidar com a morte no ofício do profissional de saúde. In: Franklin SS, organizador. Cuidados Paliativos: discutindo a vida, a morte e o morrer. São Paulo: Atheneu; 2009. p. 302-319.

26. Tallman K, Greenwald R, Reidenouer A, Pantel L. Living with advanced illness: longitudinal study of pacient, family and caregiver needs. Perm J 2012; 16(3):28-35.

27. Neergaard MA, Olesen F, Jensen AB, Sondergaard J. Palliative care for cancer patients in a primary health care setting: Bereaved relatives' experience, a qualitative group interview study. BMC Palliat Care 2008; 7(1).

28. Starfield B, organizador. Atenção primária: equilíbrio entre necessidades de saúde, serviços e tecnologia. Brasília: Organização das Nações Unidas para a Educação, a Ciência e a Cultura, Ministério da Saúde; 2002.

Artigo apresentado em 30/04/2013

Aprovado em 22/05/2013

Versão final apresentada em 17/06/2013 